\title{
Chronic Renal Failure and Aortoiliac Disease: Two Cases with Different Treatments and Outcome, and Literature Update
}

\author{
Maroun M Abou-Jaoudé ${ }^{1,2 *}$, Osama S Al Beteddini ${ }^{1}$ and Alain N Khalaf ${ }^{2}$ \\ ${ }^{1}$ Department of Surgery, Sacre-Coeur Hospital, Baabda, Lebanon \\ ${ }^{2}$ Department of Surgery, The Middle East Institute of Health, Bsalim, Lebanon
}

\begin{abstract}
Purpose: An association between peripheral arterial disease (PAD) and end-stage renal disease (ESRD) was suggested. We discuss the cases of two kidney transplant patients of different etiologies, and management outcomes.

Case Report: The first case was for a patient with long-standing complicated type 2 diabetes who suffers from advanced atherosclerotic disease involving both femoro-iliac arterial axes. The patient had successful kidney transplantation (KT) to atherosclerotic external iliac artery after endarterectomy, with a smooth early post operative course and a two-year follow up with no significant morbidity. The second case is for a patient who underwent KT, and who had previously undergone an endovascular bifurcated aortic bi-iliac stent (EVBAIS) for an infra-renal abdominal aortic aneurysm.
\end{abstract}

Conclusions: The decision concerning the presence of extensive atherosclerosis, endovascular stenting and/or endarterectomy, pre-transplantation assessment, and post-operative course will be presented. The literature is also reviewed to assess practices for detecting PAD in kidney transplant patients, and subsequent management options.

Keywords: Atherosclerosis; Endarterectomy; Kidney transplantation; Peripheral arterial disease

\section{Introduction}

Peripheral arterial disease (PAD) is a serious complication affecting successful allograft transplantation, as it is associated with widespread atherosclerotic changes affecting vascular beds [1]. An association between PAD and renal failure was recognised, since both conditions share common pathophysiological mechanisms, and are linked to significant morbidity and mortality [2]. The increase in the number of patients presenting with PAD and end-stage renal disease (ESRD) presents challenge in terms of therapeutic options, which include pre-operative revascularization, concurrent therapy or delayed revascularization [1]. Surgical interventions ranging from endarterectomy to aorto-iliac bypasses are the treatment of choice for complex lesions [1]. Advances in endoluminal arterial angioplasty and kidney transplantation (KT) have changed the course of both pathological entities $[3,4]$, improving the results of therapeutic measures to this group of patients [3]. We discuss in this paper the cases of two kidney transplant patients of different aetiologies, and as such management outcomes. The literature is reviewed to assess the therapeutic standards followed in managing both conditions.

\section{Case 1}

\section{Case summary}

A 76-year-old non-smoker Caucasian male, who has undergone in 1994 a coronary artery bypass graft for four-vessel disease, and had a bilateral external iliac artery angioplasty with stent placement in 2005 followed by a right-sided popliteal artery angioplasty with stent placement 2007. He had type 2 diabetes (T2DM) for more than 15 years. He presented in 2006 with diabetic glomerulosclerosis and drugrelated interstitial nephritis resulting in chronic renal failure. The renal function progressively deteriorated (urine output $<500 \mathrm{~mL}$ ), and was started on haemodialysis ( 3 sessions/week) via left-sided radiocephalic fistula by the end of 2008, and was referred to our institution for KT in July 2009.

Physical examination revealed body mass index (BMI) of $23 \mathrm{~kg} / \mathrm{m}^{2}$, weak pulse over the left femoral artery, and a murmur at the site of both femoral arteries. He presented with stage 1 hypertensive retinopathy, with moderate vascular tortuosity, with no evidence of haemorrhage or exudates. Treatment at the time included nitrate derivatives (corvasal cynt), beta-blocker (dilatrend), calcium channel blocker (Lercadip), clopidogrel (Plavix), simvastatin (vastarel), a-blocker (minipress), Tamsulosin (Omnic) $\alpha-1 \mathrm{~A} / \alpha-1 \mathrm{~B}$ reductase inhibitor (for prostatism), and glimepirid (Amaryl). Pre-transplant workup cleared the patient to undergo $\mathrm{KT}$.

No abnormalities in the renal vessels were detected, and cardiac ejection fraction was $45-50 \%$, mild mitral regurgitation, mean pulmonary artery pressure of $52 \mathrm{mmHg}$, and normal left ventricular function. Doppler analysis showed hemodynamically non-significant stenoses in both carotid bifurcations, but calcified atheromatous plaques through the entire length of ilio-femoro-popliteal axes, with no hemodynamic changes following angioplasty. Iliac arteries workup showed one stent extending throughout left common iliac artery from aortic bifurcation to femoral artery, a stent extending from the origin of the right common iliac artery to the iliac bifurcation, a third stent located at the distal end of the external iliac artery, leaving $8 \mathrm{~cm}$ of the external artery free, though calcified, and a fourth seen at the right popliteal artery. Hyperechogenic renal cortices with multiple cortical cysts and prostatic hypertrophy were both detected on abdominal and pelvic ultrasound. Blood testing showed elevated serum creatinine $(6.82 \mathrm{mg} / \mathrm{dL})$, microcytic anemia and normal liver function tests, thyroid profile, and acid-base status.

*Corresponding author: Dr. Maroun M. Abou Jaoude, Department of Surgery, Sacre-Coeur Hospital, Baabda, Lebanon, Tel: 961-3889977; E-mail: marounaboujaoude@hotmail.com

Received March 12, 2013; Accepted March 20, 2013; Published March 25, 2013

Citation: Abou-Jaoudé MM, Al Beteddini OS, Khalaf AN (2013) Chronic Rena Failure and Aortoiliac Disease: Two Cases with Different Treatments and Outcome, and Literature Update. J Transplant Technol Res 3: 120. doi:10.4172/21610991.1000120

Copyright: (C) 2013 Abou-Jaoudé MM, et al. This is an open-access article distributed under the terms of the Creative Commons Attribution License, which permits unrestricted use, distribution, and reproduction in any medium, provided the original author and source are credited. 


\section{Procedure}

KT was performed two days following trans-urethral prostate resection, with a smooth post-operative course. The transplant was heterotopically performed in the right iliac fossa, at the level of the right external iliac artery after endarterectomy of severely calcified artery. Intra-operative induction was initiated by 3 vials of i.v. daclizumab, and intravenous bolus of 5000 units of heparin was given prior to arterial clamping; the total vascular anastomoses was 29 minutes. The kidney produced urine immediately after removal of the vascular clamps.

\section{Course}

The post-operative renal function was excellent, with a rapid drop in serum creatinine levels. Cardiovascular protection was done by continuous intravenous perfusion of heparin (15,000 units/day), which was started $6 \mathrm{~h}$ after surgery, and continued during the hospital stay, then replaced by low-dose aspirin $(81 \mathrm{mg})$, and pravastatin upon discharge. Doppler ultrasound of the kidney graft revealed a renal resistance index of $0.58-0.67$ on day six after the surgery. As serum creatinine levels increased from $1.29 \mathrm{mg} / \mathrm{dl}$ to $1.43 \mathrm{mg} / \mathrm{dl}$ on the $6^{\text {th }}$ post-operative day, the patient was given three $500 \mathrm{mg}$ intravenous boluses of methylprednisolone, but with no improvement. Therefore, a kidney biopsy was done under CT scan guidance, and revealed no remarkable changes. Serum creatinine dropped to $1.14 \mathrm{mg} / \mathrm{dl}$ on the $10^{\text {th }}$ post-operative day, when the patient received one unit of filtrated and irradiated packed red blood cells transfusion due to low hemoglobin blood level $(6.5 \mathrm{mg} / \mathrm{dl})$. He was then discharged from the hospital on the same day in good clinical condition; treatment comprising prednisone (20 mg OD), mycophenolate mofetil (750 mg BID), and tacrolimus ( $7 \mathrm{mg}$ BID). The patient was re-admitted to the hospital on the $22^{\text {nd }}$ post-operative day for recurrent lymphocele, which was managed surgically by peritoneal fenestration.

\section{Case 2}

\section{Case summary}

The second patient is a 70 year-old Caucasian male, with essential hypertension for over 10 years, which resulted in hypertensive nephrosclerosis and stable ESRD. Following coronary artery bypass grafting (CABG) done in June 2007 for 4-vessel ischemic heart disease, with mild mitral valve regurgitation, he developed severe deterioration of renal function, which required starting hemodialysis one month after CABG. A $5.5 \mathrm{~cm}$ infra-renal abdominal aortic aneurysm (AAA) was discovered during workup for his deteriorating kidney function, and due to the occurrence of 'blue toes' syndrome due to cholesterol embolization, this was subsequently treated successfully in September 2007 by inserting an endovascular bifurcated aortic biiliac stent (EVBAIS). He was admitted in December 2007 for kidney transplantation.

The patient did not have T2DM or ischemic cerebrovascular disease, but was a heavy smoker. Physical examination was unremarkable, except for marked weight loss related to his ESRD, with normal BMI $\left(24 \mathrm{~kg} / \mathrm{m}^{2}\right)$ before the operation. Blood pressure readings were normal, and femoral and distal pulses were palpable with no clinical symptoms of arterial insufficiency, including trophic changes, peripheral edema, or pain upon exercise. Pre-transplant investigations, including cardiac workup, did not contraindicate surgery. Magnetic resonance angiogram revealed patent intra-aortic stent, ending distally at the mid common iliac arteries bilaterally, with evidence of a discrete atheroma in the descending thoracic aorta. Doppler ultrasound of the lower limbs showed good arterial perfusion, but decreased venous return.

\section{Procedure}

The transplant was performed in December 2007. The graft was installed into the right iliac fossa, using standard technique. Two arteries were identified in the kidney graft, and were reconstructed side-to-side on the back table, before doing the anastomoses with the right external iliac vessels, distal to the vascular endograft. A bolus of heparin (5000 IU) was given prior to arterial clamping just below the bifurcation of the common iliac artery (to prevent thrombosison-stent). Total vascular anastomoses time was $35 \mathrm{~min}$. The kidney produced urine immediately after removing the vascular clamps.

\section{Course}

The patient received 2 vials of daclizumab at induction of anaesthesia, to which he developed an anaphylactic shock, which required resuscitation. This lasted few hours after surgery, but responded favorably to hydration and intravenous adrenaline perfusion. The post-operative course was uneventful thereafter, and serum creatinine level had rapidly dropped to $0.7 \mathrm{mg} / \mathrm{dl}$ within 48 hours. Maintenance immunosuppression consisted of cyclosporine microemulsion, mycofenolate mofetil, and prednisone. Cardiovascular protection was done by continuous intravenous perfusion of heparin (15,000 units/day), which was started $6 \mathrm{~h}$ after surgery, and continued during the hospital stay.

Heparin was replaced by low-dose aspirin $(81 \mathrm{mg})$ and pravastatin. Echo-Doppler of the kidney graft done prior to discharge showed normal kidney graft, with a good renal resistance index of 0.69 . The patient had normal serum creatinine level $(0.7 \mathrm{mg} / \mathrm{dl})$ upon discharge, and was subsequently followed up closely in the outpatient transplant clinic. He had stable medical condition, with excellent renal function, and no vascular problems related to the endovascular stent or peripheral emboli, which have resolved with proper treatment

\section{Discussion}

Since chronic renal failure and hemodialysis are associated with hypertension and dyslipidemia - both of which predispose to accelerated atherosclerotic changes [2] - there is a steady increase in the number of patients with ESRD and advanced atherosclerosis who are candidates for kidney transplantation $[1,2,4]$. This translates into increased number of patients with ESRD in association to aortoiliac atherosclerotic and aneurysmal diseases, who require surgical and/or endovascular repair of diseased arteries [5]. Aortoiliac reconstruction is advocated as preparation for $\mathrm{KT}$ when significant atherosclerosis is noted [6,7]. Since long-term survival of kidney transplant patients was paralleled by increases in AAA development [8], this suggests that advanced ESRD needing KT, and advanced PAD requiring surgical or endovascular correction can be done either simultaneously, or alternatively after surgical or interventional treatment of the other.

Insofar as kidney transplant patients may tolerate standard open AAA repair with no protective measures for the transplanted kidney [9], renal ischemia can irreversibly damage kidney function [8]. While management of renal ischemia include axillofemoral shunting [10], and extraanatomic aortofemoral bypass [8], these increase the risk of thromboembolism at the level of the transplanted kidney [11]. The first successful endovascular repair of AAA [6], and EVBAIS placement [12], were performed in 1991 and 1994, respectively, and were described as treatments-of-choice in high-risk renal failure and kidney transplant patients [13].

An important advantage of endovascular vs. surgical repair is the relatively minimum invasiveness, highlighted by the fact that in 
Citation: Abou-Jaoudé MM, Al Beteddini OS, Khalaf AN (2013) Chronic Renal Failure and Aortoiliac Disease: Two Cases with Different Treatments and Outcome, and Literature Update. J Transplant Technol Res 3: 120. doi:10.4172/2161-0991.1000120

endovascular repair, the aorta is not clamped, and thus blood loss is less. However, this technique has some shortcomings, namely that patients managed with endovascular repair may develop long-term complications due to endovascular graft failure, such as endoleak and graft migration, thus necessitating routine follow-up [5]. On the other hand, endovascular repair minimises the visceral ischemia time resulting in lower mortality rates. This is of direct relevance to kidney transplant recipients presenting with atherosclerotic or aneurysmal disease in need of repair, as it offers the possibility of preserving the functioning allograft. The rapid evolvement of technologies in endovascular repair has resulted in wide adoption of this technique as a standard management in these high risk patients [14].

Aortoiliac atherosclerosis is frequently seen in renal failure patients on the transplant waiting list, which questioned whether angiography should be routinely performed in patients over 40 years [6]. In our program we routinely perform angiography, Angio scan or Angio magnetic resonance imaging of the aorta, iliac vessels and the peripheral vascular system in all atherosclerotic, or diabetic patients as well as in all potential recipients above 50 years of age. We believe also that the routine administration of intravenous heparin $6 \mathrm{hrs}$ after KT followed by the use of low-dose aspirin $(81 \mathrm{mg})$ and pravastatin might prevent any vascular complication on long term. Vascular reconstruction may be performed before transplantation, when arterial reconstruction is performed by prosthetic grafts, and the recommended interval for transplantation is 6 weeks to 3 months. Alternatively, arterial reconstruction (performed by either endarterectomy or construction of a bypass using an artificial vascular prosthesis or arterial allograft) and KT may be performed simultaneously [15]. The risk of infection associated with the conduit is fairly high, especially in cases where a urinary fistula develops after the transplantation procedure. As such, a fresh arterial allograft is a safer option, and its manipulation is easier [15].

\section{Conclusion}

Chronic renal failure and aortoiliac atherosclerotic and aneurysmal disease share a common pathophysiological mechanism. With an increasing population of patients presenting with these entities, several challenges are awaiting transplant and vascular surgeons and interventional radiologists. A multi disciplinary approach is highly recommended to draw the best treatment strategies for getting better outcomes in the short- and long-terms aiming at decreased mortality rates in this particularly high-risk group.

\section{References}

1. Gill R, Shapiro R, Kayler LK (2011) Management of peripheral vascular disease compromising renal allograft placement and function: review of the literature with an illustrative case. Clin Transplant 25: 337-344.

2. Tian SL, Murphy M, Han QF, Lu XH, Wang T (2010) Prevalence and risk factors for peripheral artery disease among patients on maintenance peritoneal dialysis. Blood Purif 30: 50-55.

3. Ridgway D, White SA, Nixon M, Carr S, Blanchard K, et al. (2006) Primary endoluminal stenting of transplant renal artery stenosis from cadaver and nonheart-beating donor kidneys. Clin Transplant 20: 394-400.

4. Valpreda S, Messina M, Rabbia C (2008) Stenting of transplant renal artery stenosis: outcome in a single center study. J Cardiovasc Surg (Torino) 49: 565570 .

5. Kokotsakis J, Kaskarelis I, Koukoulaki M, Athanasiou T, Skouteli E, et al. (2009) Entire stent grafting of thoracoabdominal aorta in a renal transplant recipient subsequent to extra-anatomical bypasses of the main abdominal vessels. Ann Thorac Surg 87: 623-625.

6. Brekke IB, Lien B, Sødal G, Jakobsen A, Bentdal O, et al. (1993) Aortoiliac reconstruction in preparation for renal transplantation. Transpl Int 6: 161-163.

7. Lacombe M (2008) Surgical treatment of aortoiliac aneurysms in renal transplant patients. J Vasc Surg 48: 291-295.

8. Ailawadi G, Bedi A, Williams DM, Stanley JC, Upchurch GR Jr (2003) Endovascular treatment of aortic aneurysms in renal transplants. J Vasc Surg 37: 693-696.

9. Jivegård L, Blohmé I, Holm J, Karlberg I (1989) Abdominal aortic reconstruction without renal bypass in renal transplant patients. Surgery 106: 110-113.

10. Schwartz RA, Caracci BF, Peterson GJ (1988) Successful repair of a ruptured abdominal aortic aneurysm in a renal transplant recipient. Ann Vasc Surg 2: 189-192.

11. Botta GC, Capocasale E, Rubini P, Tondo S (1999) Resection of an aortic aneurysm in a renal transplant recipient using hypothermic perfusion. Nephrol Dial Transplant 14: 1295-1296.

12. Scott RA, Chuter TA (1994) Clinical endovascular placement of bifurcated graft in abdominal aortic aneurysm without laparotomy. Lancet 343: 413.

13. Greenhalgh RM, Powell JT (2008) Endovascular repair of abdominal aortic aneurysm. N Engl J Med 358: 494-501.

14. Haulon S, O'Brien N (2011) Advances in endovascular repair for complex aneurysms. Br J Surg 98: 163-165.

15. Adamec M, Janouöek L, Toöenovský P, Lácha J (2000) Renal transplantation combined with aortofemoral bypass using a fresh arterial allograft. Transpl Int 13 Suppl 1: S56-S59. 